\title{
REVIEW PAPER ON DIGITAL MANUFACTURING \& DESIGN
}

\author{
Mukhtiar Singh \\ Department of Mechanical Engineering, \\ Lovely Professional University \\ Jalandhar - Delhi G.T. Road, Phagwara, Punjab 144411 \\ mukhtiarts@gmail.com
}

\begin{abstract}
The transformation is happening through digital manufacturing and design (DM\&D) - a shift from paper-based processes to digital processes in the manufacturing industry.as technology is having a huge impact on the ways products or services are designed \& manufactured. Ever increasing customer demands for newer products with better features is transforming the dynamic of manufacturing towards embracing digital technology. Mass customization of products and services is preferred over mass production. There is an increased pressure upon businesses to serve an individual customer at a competitive cost comparable to the mass production cost, with shortest possible development time and production by focusing on levels of customization, quality and performance along with competitive price and value. With the advancements made in Information Technology, Material Science, Production Technologies and Supply Chain Strategies for the past 50 years' manufacturers are well positioned to challenge the traditional methods the products are designed and manufactured. Digital manufacturing and design encompasses visualization, manufacturing simulation, ergonomic and human factor analyses, holistic view of product and process design, development and product design sensitive to the process constraints and capabilities. The next stages of the new era and next Industrial Revolution termed as INDUSTRY 4.0. This article is all about defining a transformational shift from conventional methods of manufacturing to Digital Manufacturing adopting factors like Digital Thread, Advanced Analysis, Intelligent Machining, Security in Manufacturing, Model based system Engineering and reconfigurability in manufacturing systems etc
\end{abstract}

Keywords- Digital Manufacturing \& Design, Digital Thread, Intelligent Machining, Digital Manufacturing Security, Cyber Security, Model Based System Engineering

\section{INTRODUCTION}

The manufacturing environment radically changed over the last decades as manufacturing companies strived to improve their performance in a globalized, interconnected and volatile market environment. Manufacturing companies in the 21 st century face increasingly frequent and unpredictable market changes driven by global competition, including the rapid introduction of new products and constantly varying product demand. Traditional approaches of dedicated production lines, although still in use/practice, are becoming irrelevant with changing market requirements such as; increases in product demand, product changes, such as the introduction of a new product in the line, and system failures, such as a machine breakdown, in a cost effective or timely manner. In a highly competitive market and high volume manufacturing, such as automotive, the introduction of automation and information and communication technologies (ICT) on the shop floor, supported by digital manufacturing tools, has led to more flexible production systems which are capable of dealing with the volatile market demands and having a mixed product flow Digital Manufacturing \& Design is a platform for exploring new methods for manufacturing product. Digital manufacturing evolved from manufacturing practices such as design for manufacturing(DFM), flexible manufacturing (FM), lean manufacturing (LM) and computer-integrated manufacturing (CIM) which requires the collaboration across process and product design. The first Industrial Revolution, began in mid-1800s represented the transition from manual production to mechanical production, empowered by outfitting of water and steam power control [1]. The Second Industrial Revolution came at the beginning of the $20^{\text {th }}$ century with the advancement of methodologies that empowered large scale manufacturing, including the mechanical production system [2]. The Third Industrial Revolution, otherwise called Digital Revolution, represented the advances made conceivable via robotization as mechanical and simple advances offered approach to computerized gadgets between the 1950s and the 1970s. The Fourth Industrial Revolution which is also called Industry 4.0 is the succeeding stage in the development of manufacturing.

The cyber capabilities resulting from advances in computing combined with physical systems to have enabled a highly intelligent, SMART (Sustainable Manufacturing and Advanced Robotics Technologies) machines that integrates design, and produces robotized manufacturing ecosystem [3]. The Formal definition of Manufacturing is the way towards changing over the crude materials, segments, or parts into completed products that meet a client's desires. Manufacturing is the most tangible part of the product lifecycle because it results in a clear outcome [4]. 
In recent years, industrial nations around the globe have invested heavily in new technologies, software, and services to advance digital design and manufacturing using cyber-physical systems, data analytics, and highperformance computing for optimum product and process design. In product life cycle stages, design begins in an abstract fashion with conceptual design and concludes with detail design, where final detailed part specifications are created. The subsequent step of the process is to take the product from a design and develop it. This stage includes defining the items that need to be manufactured and how they will be created and assembled. In product design and development process, production prototypes are created and checked to see if they meet performance and cost requirements. As well as long term production capabilities are established [5]. Next stage is support stage where once produced and purchased by the customer, the product must still be supported. The support stage of the lifecycle is where you provide the customer with information to assist in the effective use of the product and maintain the operational performance of the product. Product's lifecycle ends with disposal and recycling. The dispose state of the lifecycle draws on information from the design and build phases to understand the composition and what portions of a product can be recycled into future applications [6]. The dispose stage may also play a crucial role in iterating the design to further develop a product that meets performance expectations and that can be disassembled at the end of life. With the possibilities of many iterations, multiple opportunities for improvement have been identified for traditional design and manufacturing processes over the time. In a product's lifecycle, many iterations may be required before customer approval is received.

There are various challenges associated with traditional paper bases processes as in paper based processes, information sharing can consume valuable time and delay the development process. In traditional processes, there is also a lag during and in- between stages in the lifecycle as necessary approvals are sought [7]. Sometimes, this can even result in losing first-tomarket competitive advantage. Another challenge in paper based processes is that maintaining a single source of true information for product and process definitions is very difficult and can limit the capabilities for data analysis and evidence based decision-making. Frequent communication gaps can result when planned changes are made during design and development process, as well as after the production launch. To address all these issues a new approach called digital manufacturing is most appropriate. The concept of digital manufacturing is an integrated suite of tools that work with product definition data to support tool design, manufacturing process design, visualization, modelling and simulation, data analytics, and other analyses necessary to optimize the manufacturing process as this approach uses advanced computing power to optimize the product lifecycle. [8]. Digital manufacturing allows you to more efficiently and commandingly share information in the design process.
The term Digital thread refers to the communication framework that allows a connected data flow and integrated view of the asset's data throughout its lifecycle across traditionally siloed functional perspectives can be used to create a computer based digital twin which is an integrated system of data, models, and analyses that can be used in design, manufacturing, support, and disposal. The concept of digital thread is very useful in designing smart manufacturing which strives to orchestrate and optimize business, digital and physical processes across factories and the entire product value chain. The digital thread also provides a formal framework for controlled interplay of authoritative technical and as-built data with the ability to access, integrate, transform and analyze data from disparate systems throughout the product lifecycle into actionable information. This whole idea is called as the Fourth Industrial Revolution, Industry 4.0 [9].

\section{PRODUCT LIFECYCLE \& CHANGE TRANSITIONED}

Product lifecycle refers to all the stages of a product concept design to disposal and recovery. Product lifecycle can be seen from different points of view. All in all, item lifecycle covers three primary stages: start of life, including outline and assembling; center of life, including use, administration, and upkeep; at last the end of life, these products are re-collected, arranged, dismantled, re-manufactured, reused, \& disposed [10]. Different organization have different motivations to collect product lifecycle data. A couple of them are transparency, business assessment, change, and estimating. Full transparency empowers both the purchasers and producers to settle on better educated choices [11]. Cases of transparency incorporate the arrival of the nourishment data on sustenance items and the arrival of natural effects data on products to customers. With a specific end goal to have transparency, we have to gather and offer information of various parts of an item or venture. Another motivation to collect product lifecycle data is to use data for business evaluation and improvement [12]. Continuous improvement requires continuous evaluation of the business process from different aspects. Continuous evaluations require continuous collection of data to evaluate [13]. By collecting data, the enterprise management is able to forecast valuable information. An example of this is when an enterprise collects the market trends to predict future market demands. Another is when an enterprise collects the energy usage of an industrial machine to predict its failure. As we just learned, the product life-cycle covers three main phases: beginning of life, middle of life, and end of life. The information flow during the beginning of life is quite complete and is collected using different information management systems, such as computer-aided design, or CAD models, computer-aided manufacturing, or CAM systems, and product data management or PDM systems [14]. The information flow becomes less complete after the beginning of life cycle. In fact, the information flow is stopped after product is delivered to a consumer for the majority of today's consumer products such as household 
appliances and vehicles. This is because after products are delivered to consumers, the ownership of product is no longer with companies and is transferred to customers, therefore, it is not easy for companies to track product usage data generated by consumers. As a result, decision makers involved in each phase of a product lifecycle make decision based on an incomplete and inaccurate product lifecycle data from other phases, which in many cases result into inefficient decisions. Product lifecycle data can be categorized under static data and dynamic data [15]. Static data is mainly generated at the beginning of life phase and rarely changes during the lifetime of the product. Examples of static data are bill of materials, material content, take back information, disassembly instruction, return policies, and recycling information. Static data is fairly complete and can be collected through existing data management systems. The dynamic information incorporates mainly the information created during usage phase. Cases of progression information which utilize designs, natural conditions, and adjusting activities [16]. Dynamic information is regularly lost and is hard to acquire during product lifecycle. Product lifecycle have numerous applications. Assume that organizations can track and follow product utilization information produced by buyers \& consumers [17]. Companies could gain substantial business advantages if they use product usage data to improve their products and optimize relevant operations [18]. For example, lifecycle acquisition can be used to enable productrelated services. Product lifecycle data is valuable and can be used to facilitate the service delivery such as maintenance, repair, and after-sales services. It is particularly important in the aviation industry. In aviation industry, capital equipment, and products have long service lives and complex configurations [19]. The productivity of the business is not just from the offers of capital gear and flying machines, yet from keeping up them for an expected 30 or more-year life expectancy. Along these lines, upkeep and repair organizations mean to limit support cost and turnaround time to boost income. Because of the many-sided quality of the framework, robotized data recovery and item structure data can help organizations effortlessly recognize their potential disappointments and acquire the data essentially for part repair and tooling plan. Gathering item lifecycle information is a spine for an incorporated enterprise.

Data standards are very useful to establish a relationship between two different datasets to create information or connect machines from different manufacturers. They specify a consistent data format so that the data can be shared and transformed into usable information. Some examples are, STEP and IGES, are data standards for sharing product design data like geometry [20]. MT Connect is a standard that supports the capture and sharing of information from machining equipment. Mil Standard 31000A and A SME 1441 define ways to share product model information using a technical data package to support the product life cycle.

\section{DIGITAL THREAD}

From the initial concept, products evolve through a regular sequence of plan, design, build, support, and dispose. During each of these stages, a colossal measure of information is produced. Although traditionally, much of it has not been captured, it can be processed into very valuable information that can impact the product life cycle in many different ways. A name's been given to describe all of the data generated in every part of a product's life cycle- the digital thread. The digital thread concept seamlessly integrates information through the value chain from requirements gathering through feasibility reviews, design, manufacturing, testing, and on to final sustainment and disposal phases. This means specialists, throughout the process, can work on the product, and process definitions simultaneously to inform decisions, through the life of a system or product. While promising, adoption of the digital thread was initially limited, but has continued to gain momentum. Like any paradigm shift, the digital thread approach is potentially disruptive, especially to organizations that do not have in house information technology resources [21]. For example, the information technology foundation for the digital thread has typically been viewed as overhead, simply a cost of doing business that needs to be shortened.

The digital thread paradigm views the IT infrastructure as an investment that will generate a return in increased effectiveness and responsiveness. A second challenge, facing the digital thread, is the transitioning profile of manufacturing jobs from skilled to smart or information workers that combine their expertise with physical systems, and the ability to work with the data generated in the production process [22]. This change will more tightly link production staff with the design process. Giving them more opportunities to impact the design of a product that they create. A third challenge facing the digital thread implementation is natural. How do we keep the information secure? Traditional processes used physical documents that could be controlled and locked up. There's a natural resistance to putting valuable intellectual property in digital form, especially as retailers seemingly report intrusions on a regular basis. Information security is a key component of the digital thread, and as we all see, is incorporated from the beginning [23]. Sharing data and information between different functions and systems of the product life cycle can add significant value to the entire development process. Some examples are, faster product launches, clearer communication between suppliers and manufacturers, resulting in fewer errors, reduced rework and scrap. Reduced inventory in the supply chain. Reduced unit product cost which increases profit margin. And even the expansion to additional markets.All around, the techniques by which products are composed and fabricated are progressing at a quick pace just directly in front of us. Traditionally processes, checked and controlled by people, are presently being controlled and observed by brilliant sensors and actuators in this new era of manufacturing [24]. 
Intelligence of a system can be perceived as the capability of the system to achieve a goal or to sustain desired behavior in business of uncertain conditions. In context of manufacturing, intelligent machining is a criterion in which machine tools can identify their own states and the environment in which they are operating, and can execute start, control, and terminate machine activities [25]. In other words, intelligent machines are self-worth and can make decisions related to assembling processes. The intelligent machining worldview is empowered to the coordination of keen sensors and controls. Advances in sensor technologies and automated data systems, provide new opportunities for real time collection of valuable measurements that can be converted into actionable data to make smart decisions [26].

The data acquisition and management module is comprised of the data aggregator component, a data post processor, and a data buffer component. The data aggregator component collects time synchronized raw data from external sensors. Since the data generated from different sensors can be in different formats, they need to be transformed into single format to enable it to be processed into information. Data aggregators allow for this interoperability [27]. Empty connect, is an example of industry standard data aggregation agent that allows for data aggregation. Data processor processes the collected data into suitable data formats that can be used by machine learning algorithms to make smarter decisions. Whereas, data buffer allows for data buffering so that the unwanted data can be erased at this point. The data buffer stores only relevant data that could be used by machine learning algorithms to generate actionable intelligence. Data buffers act where in between the data processing and machine learning algorithms and allows for efficient storage [28]. The data dividend machine learning and knowledge discovery module, uses the processed monitoring data to construct a data dividend prediction model for the target machine. The prediction model is used in conjunction with the data from past experiences to monitor and control the machining tool.

\section{ADVANCED MANUFACTURING ENTERPRISE}

According to the National Institute of Standards and Technology, advanced manufacturing is an entity that makes extensive use of computer, high precision and data innovation incorporated with a superior workforce in a creation framework equipped for outfitting a heterogeneous mixture of products in little or huge volumes with both the productivity of large scale manufacturing and the adaptability of custom assembling so as to react quickly to client demands. Anderson Economy Group has defined has defined advanced manufacturing as, operations that create advanced products, use innovative techniques in their manufacturing, and are inventing new processes and technologies for future manufacturing [29]. Advanced Manufacturing is not quite recently the work of robots and cutting-edge forms yet in addition incorporates the assembling of imaginative items and utilization of innovative standards. Components of cutting edge producing are arranged under two primary classes, tangible and intangible components. Tangible elements of manufacturing systems include manufacturing processes, industrial control systems, industrial robotics, assembly systems, material transportation systems and storage systems. Intangible elements of assembling frameworks incorporate all arranging and administration part of the assembling firm, cases of cutting edge innovations incorporate Nano-fabricating, semiconductor, additive manufacturing and bioproducing.

\section{DIGITAL MANUFACTURING SECURITY}

Digital manufacturing via the digital thread requires the consideration of security in all domains including network, desktop, hardware, and software applications. Security is, in fact, an integral part of the overall digital manufacturing design since it involves various valuable intellectual property resources, which we call IP throughout this course [30]. And should not be confused with IP, an acronym used in computer networks to mean Internet protocols. These IP resources are mostly information such as hardware designs, machine control instructions, payroll transactions, or business deals. The leak of IP information can cause huge loss to your company. And therefore, IP needs primary attention while designing system security. Threats to IP, come from various sources that includes business competitors and state affiliated actors. The biggest threats to IP is the insider threat. Since insiders are the people with legitimate access to important information and hence, such threats are hard to detect. Therefore, in DMD security, we need different levels of security to provide restricted access to the information. Such access control will allow only certain authorities in the organization to access the IP information. Recent developments in digital manufacturing may be categorized into two major groups. The developments of the first group have followed a bottom-up approach considering digital manufacturing, and extending its concepts, within a wider framework e.g. the digital factory or enterprise. The developments of the second group have followed a top- down approach considering the technologies in sup- port of individual aspects of digital manufacturing, e.g. e-collaboration and simulation. The digital factory includes models, methods, and tools for the sustainable support of factory planning and factory operations. It includes processes based on linked digital models connected with the product model. At a theoretical level, several researchers have contributed to the definition of the digital factory vision and suggested how this vision could be implemented in reality. From a technological point of view, new frame-works for distributed digital manufacturing have appeared on the scene. Recent developments focus on a new generation of decentralized factory control algorithms known as 'agent based'. A software agent, first, is a self-directed object, second, has its own value systems and a means of communicating with other such objects, and, third, continuously acts on 
its own initiative. A system of such agents, called a multi-agent system, consists of a group of identical or complementary agents that act together. Agentbased systems encompassing real-time and decentralized manufacturing decision-making capabilities have been reported.

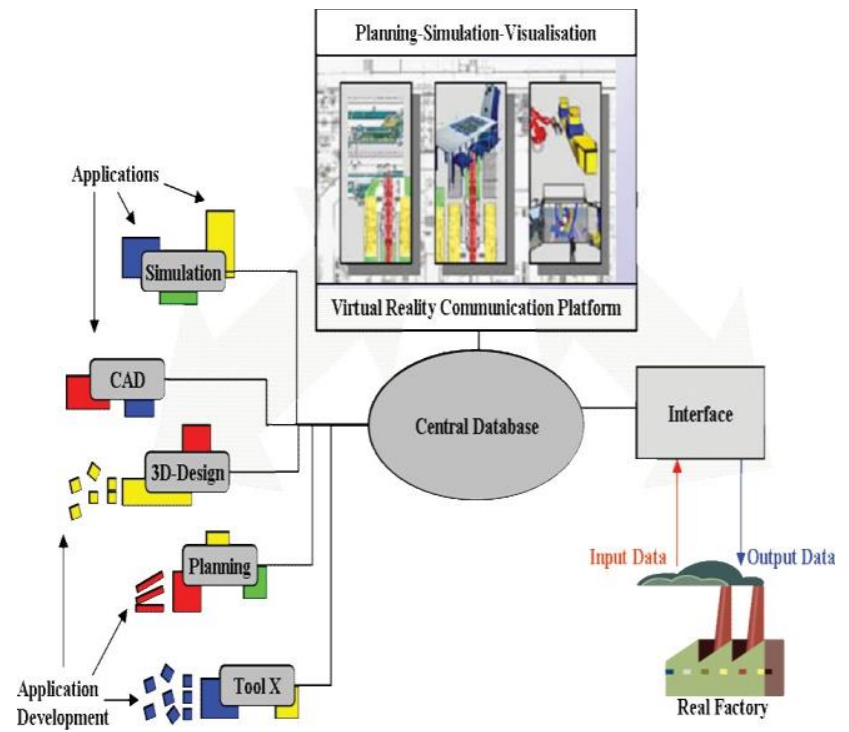

The vision of the digital factory

\section{MODEL BASED SYSTEM ENGINEERING}

The INCOSE systems engineering handbook points to three meaningful definitions of systems engineering [31]. A complete definition - Systems Engineering is an integrative approach which intends to empower the recognition of acknowledged systems. It focuses on characterizing client needs and required outputs, in the development cycle. Documenting stipulations and then proceeding with design composites and system demonstration while considering the complete problem [32]. Operations, cost and schedule, performance, training and support, test, manufacturing and disposal [33]. It accommodates all the disciplines and specialty groups into a team effort framing a structure advancement process that proceeds from idea, to production, to operation. It additionally considers both the business and industrial needs of all clients with the goal providing a quality product that meets the consumer needs.

Systems engineering is a reiterative process of top down synthesis, reinforcement, and operation of a realworld system that satisfies, in near optimal manner, the full range of requirements for the system. From an operational perspective, System engineering is a development engineering that scrutinize on the design and operation of the entire framework as particular from the parts.

\section{A. System Engineering \& Lifecycle}

A system progresses through its life cycle as the result of actions. Performed and managed by people in organizations, using processes for execution of their actions. The life cycle stages used the standard are concept, development, creation, usage, support and retirement. Different models have emerged for managing the processes within and across life cycle stages [34]. The first life cycle model for systems engineering that is the waterfall model. The waterfall model is a specified, sequential process that follows a predetermined sequence. Waterfall is easily converted into schedules with deliverables, where the process flows downwards through all the stages that are required, concept, development, production, utilization, support and retirement. By its nature, in waterfall there is an embedded assumption that requirements are well understood and not likely to change during the execution of a project. The second approach is actually a class of methods. Incremental and iterative methods. Incremental and iterative methods have proven to be successful for small, simpler systems or elements of a system due to their focus on flexibility and judicious risk taking.

\section{B. Model-Based Definition}

According to INCOSE, model-based systems engineering, also known as MBSE, is the formalized application of modeling to support system requirements, design, investigation, confirmation, and approval exercises starting in the reasonable outline stage and proceeding through advancement and later life cycle stages. Model-based systems engineering is still in a dynamic state of development and is a vast topic to fully address. As a result, this course is offering a broad perspective with connections throughout the course that give you the opportunity to learn more about a topic of interest. A key component of model-based systems engineering is the model-based definition, or MBD. Model-based definition embodies the concept of moving away from paper-based documentation and drawings to digital, 3D CAD representation, manufacturing data, and performance models. An example of the dynamic state of MBSE, the definition of MBD is still evolving and converging. Standards, like ASME Y 14.41, ISO 16792 , and MIL-STD-31000, can provide guidance on how to annotate drawings. But multiple perspectives exist in regards to what makes up a model. The framework to use a model-based system a framework is required provides the guiding structure to implement analysis throughout the lifecycle of the product. An analysis framework connects a variety of simulation tools -- for example, Ansys, Nastran, MATLAB, and tools built in-house-in such a way that outputs of one analysis provide the input for subsequent analyses without human intervention. The Model-Based Systems Engineering (MBSE) framework is Siemens offering HEEDs. HEEDs provide a means of computationally exploring possible design alternatives in search of specific performance goals.

VII. Cloud-based Design and Manufacturing 
Cloud-based design and manufacturing (CBDM) refers to a service-oriented networked product development model in which service consumers are able to configure products or services and reconfigure manufacturing systems through Infrastructure-as-a-Service (IaaS), Platform-as-a-Service (PaaS), Hardware-as-a-Service (HaaS), and Software-as-aService (SaaS) [35]. In the IaaS model, cloud service providers offer on-demand access to computing resources such as virtual machines and cloud storage. In the PaaS model, cloud service providers deliver computing platforms such as social collaboration platforms, programming and execution 4 environments for cloud computing. In the HaaS model, cloud service providers and consumers are enabled to rent and lease manufacturing equipment such as milling machines and 3D printers. In the SaaS model, cloud service consumers are enabled to run computationally expensive application software such as AutoCAD remotely without installing and running the software on their local computers [36]. Cloud-Based Design (CBD) refers to a networked design model that leverages cloud computing, service-oriented architecture (SOA), Web 2.0 (e.g., social network sites), and semantic web technologies to support cloud-based engineering design services in distributed and collaborative environments. Cloud-Based Manufacturing (CBM) refers to a networked manufacturing model that exploits on-demand access to a shared collection of diversified and distributed manufacturing resources to form temporary, reconfigurable production lines which enhance efficiency, reduce product lifecycle costs, and allow for optimal resource allocation in response to variable-demand customer generated tasking

\section{CONCLUSIONS}

Digital Manufacturing \& Design creates a product at speed beyond human imagination. The DMD domain is inspired by a detailed thread of product, handled information and data, which brings about significant protected innovation. Industries can reduce their paper based processes to digital process which at last will lead in increase in productivity. When development of product was made initially in 8 to 9 months now had been reduced to 20 to 30 days and this is digital manufacturing. DMC will become a platform used by millions of people daily. Users can easily add their own manufacturing recipes and examine their works in their own web browsers. The digital thread is networked at several functions and levels in order to enhance efficiency and effectiveness.

DMD reduces the effects on the supply chain by transparently sharing efficiency information, whether man, machine, materials, or methods, throughout the supply chain. Increasing supply chain confidence between tiers, reducing excess inventory that's contained within the supply chain, and allowing for realizing more efficient and agile supply chain models. Data analytics ideas have taken into account the development of new assembling ideal models including the smart manufacturing, social assembling and cloud based manufacturing. Digital manufacturing incorporates technologies for the virtual representation of factories, buildings, resources, machine systems equipment, labor staff and their skills, as well as for the closer integration of product and process development through modelling and simulation Closing the gap between the product definition and the actual manufacturing production activities within the enterprise, fully transforming tacit manufacturing knowledge into tangible, and, finally, digital knowledge, optimizing data management, and developing standard models are some key priorities.

\section{REFERENCES}

[1] Brugo, T., Palazzetti, R., Ciric-Kostic, S., Yan, X. T., Minak, G., \& Zucchelli, A. (2016). Fracture mechanics of laser sintered cracked polyamide for a new method to induce cracks by additive manufacturing. Polymer Testing, 50, 301-308. doi: 10.1016/j.polymertesting.2016.01.024

[2] Capel, A. J., Edmondson, S., Christie, S. D., Goodridge, R. D., Bibb, R. J., \& Thurstans, M. (2013). Design and additive manufacture for flow chemistry. Lab on a Chip, 13(23), 4583- 4590.

[3] Choi, S., Jun, C., Zhao, W. B., \& Noh, S. D. (2015). Digital Manufacturing in Smart Manufacturing Systems: Contribution, Barriers, and Future Directions. In Advances in Production Management Systems:Innovative Production Management Towards Sustainable Growth (Vol. 460, pp. 21-29).

[4] Choi, S., Kim, B., \& Noh, S. (2015). A diagnosis and evaluation method for strategic planning and systematic design of a virtual factory in smart manufacturing systems. International Journal of Precision Engineering and Manufacturing, 16(6), 1107-1115. doi:10.1007/s12541-015-0143-9

[5] Cotteleer, M., Holdowsky, J., \& Mahto, M. (Singersongwriters). (2014). The 3D opportunity primer. On:Deloitte University Press.

[6] Coykendall, J., Cotteleer, M., Holdowsky, J., \& Mahto, M. (2014). 3D opportunity in aerospace and defense: Additive manufacturing takes flight. A Deloitte series on additive manufacturing, 1.Ding L, Davies D, McMahon CA. The integration of lightweight representation and annotation for collaborative design representation. Res Eng Des 2009; 20(3):185-200.

[7] Dehoff, R. R., Tallman, C., Duty, C. E., Peter, W. H., Yamamoto, Y., Chen, W., \& Blue, C. A. (2013). Case study: additive manufacturing of aerospace brackets. Advanced Materials and Processes, 171(3).

[8] Delaporte, P., \& Alloncle, A. P. (2016). INVITED Laserinduced forward transfer: A high resolution additive manufacturing technology. Optics and Laser Technology,78,33-41.doi: 0.1016/j.optlastec.2015.09.022

[10] Jovanovic, V., \& Hartman, N. W. (2013). Web-based virtual learning for digital manufacturing fundamentals for automotive workforce training. International Journal 
of Continuing Engineering Education and Life Long Learning XIV, 23(3-4), 300-310.

[11] Keating, L. (2014). You can now print edible pizza with a 3D 'Foodini' printer TECH TIMES. Retrieved from http://www.techtimes.com/articles/19630/20141106/star tup-serves-up-edible- pizza-burgers-3dfoodiniprinter.htm

[12] Kim, H., Lee, J.-K., Park, J.-H., Park, B.-J., \& Jang, D. S. (2002). Applying digital manufacturing technology to ship production and the maritime environment. Integrated Manufacturing Systems, 13(5), 295-305

[13] Kim Porter, Jarrod Phipps, Adam Szepkouski, \& Abidi, S. (Singer-songwriters). (2015). 3D opportunity serves it up: Additive manufacturing and food. On: Deloitte University Press

[14] Kitson, P. J., Glatzel, S., Chen, W., Lin, C. G., Song, Y F., \& Cronin, L. (2016). 3D printing of versatile reaction ware for chemical synthesis. Nature Protocols, 11(5), 920-936. doi:10.1038/nprot.2016.041

[15] Kurzrock, R., \& Stewart, D. J. (2016). Click chemistry, 3D-printing, and omics: the future of drug development. Oncotarget, 7(3), 2155-2158. Retrieved from <Go to ISI $>$ ://WOS:000369951800001

[16] Li BH, Zhang L, Wang SL, Tao F, Cao JW, Jiang XD, et al. Cloud manufacturing: a new service-oriented networked manufacturing model. Computer Integrated Manufacturing Systems 2010;16(1):1-7.

[17] Monostori L, Váncza J, Kumara SR. Agent-based systems for manufacturing. CIRP Ann 2006;55(2):697720.

[18] Piller F, Vossen A, Ihl C. From social media to social product development: the impact of social media on cocreation of innovation. Unternehmung 2012;66(1):7.

[19] Red E, French D, Jensen G, Walker SS, Madsen P. Emerging design methods and tools in collaborative product development. J. Comput. Inf. Sci. Eng. 2013; 13(3):031001.

[20] Red E, Holyoak V, Jensen CG, Marshall F, Ryskamp J, $\mathrm{Xu}$ Y. v-CAx: a research agenda for collaborative computer-aided applications. Computer Aided Design and Applications 2010;7(3):387-404.

[21] Schaefer D, editor. Product development in the sociosphere: game changing paradigms for 21 st century breakthrough product development and innovation. London, UK: Springer; 2014. p. 235.

[22] Shen W, Hao Q, Yoon HJ, Norrie DH. Applications of agent-based systems in intelligent manufacturing: an updated review. Adv Eng Inform 2006;20(4): 415-31

[23] Sutherland IE. Sketch pad a man-machine graphical communication system. In: Proceedings of the SHARE design automation workshop. ACM; 1964. p. 6-329.

[24] Tolio T, Ceglarek D, ElMaraghy HA, Fischer A, Hu SJ, Laperrière L, et al. SPECIES - co- evolution of products, processes and production systems. CIRP Ann 2010; 59(2):672-93

[25] Ulrich KT, Eppinger SD. Product design and development, vol. 384. New York: McGraw- Hill; 1995.

[26] Wu D, Thames JL, Rosen DW, Schaefer D. Enhancing the product realization process with cloud-based design and manufacturing systems. Trans ASME J Comput
Inform $\quad$ Sci $\quad$ Eng $2013 ; 13(4)$ : http://dx.doi.org/10.1115/1.4025257. 041004-04100414

[27] Wang L, Shen W, Lang S. Wise-ShopFloor: a web-based and sensor-driven e-shop floor. Trans ASME J Comput Inform Sci Eng 2004;4(1):56-60.

[28] Wu D, Rosen DW, Schaefer D. Cloud-based design and manufacturing: status and promise.In: Schaefer D, editor. Cloud-based design and manufacturing: a serviceoriented product development paradigm for the $21 \mathrm{st}$ century. London, UK: Springer; 2014. p. 282

[29] Wu D, Greer MJ, Rosen DW, Schaefer D. Cloud manufacturing: strategic vision and state-of- the-art. J Manuf Syst 2013.

[30] Wang L, Shen W, Xie H, Neelamkavil J, Pardasani A. Collaborative conceptual design - state of the art and future trends. Computer Aided Des 2002;34(13): 98196.

[31] Wang L, Shen W. DPP: an agent-based approach for distributed process planning. J Intell Manuf 2003;14(5):429-39.

[32] Wu D, Rosen DW, Schaefer D. Modeling and analysing the material flow of crowdsourcing processes in loud-based manufacturing systems using stochastic petri nets. In: Proceedings of the ASME 2014 international manufacturing science and engineering conference (MSEC14), Paper Number: MSEC2014-3907, Detroit, Michigan, US, 2014.http://www.compositesworld.com/cdn/cms/bey ond-big-data-harnessing-the-industrial- internet-forwind-power-5-638.jpg

[33] Yim S, Rosen DW. Build time and cost models for additive manufacturing process selection. In: Proceedings of the ASME 2012 international design engineering technical conference \& computers and information in engineering conference, IDETC/CIE12, Paper Number: DETC2012-70940, Chicago, US, 2012.

[34] Giffi, C., Gangula, B., \& Illinda, P. (2014). 3D opportunity for the automotive industry: Additive manufacturing hits the road. On: Deloitte University Press, May

[35] Manyika, J., Chui, M., Bughin, J., Dobbs, R., Bisson, P., and Marrs, A., 2013, "Disruptive technologies: advances that will transform life, business, and the global economy," McKinsey Global Institute San Francisco, CA.

[36] Wu, D., Rosen, D. W., and Schaefer, D., 2014, "Cloudbased design and manufacturing: status and promise," In: Schaefer, D. (Ed): Cloud-based design and manufacturing (CBDM): a service-oriented product development paradigm for the 21 st century, Springer, London, UK, ISBN 978-3-319-07398-9, pp. 1-24 